\title{
Modelling Rod-like Flexible Biological Tissues for Medical Training
}

\author{
Jian Chang, Junjun Pan, Jian J. Zhang \\ The National Centre for Computer Animation, The Media School, Bournemouth University, \\ Poole, BH12 5BB, U.K. \\ \{jchang, pjunjun, jzhang\} @bournemouth.ac.uk
}

\begin{abstract}
This paper outlines a framework for the modelling of slender rod-like biological tissue structures in both global and local scales. Volumetric discretization of a rod-like structure is expensive in computation and therefore is not ideal for applications where real-time performance is essential. In our approach, the Cosserat rod model is introduced to capture the global shape changes, which models the structure as a one-dimensional entity, while the local deformation is handled separately. In this way a good balance in accuracy and efficiency is achieved. These advantages make our method appropriate for the modelling of soft tissues for medical training applications.
\end{abstract}

Keywords: Medical training, surgery simulation, deformation, Cosserat rod, computer animation

\section{Introduction}

Virtual simulation can replicate the teledisplay and instrumentation of laparoscopic surgery and is attracting increasing attention from both the research community and the medical practitioners. As a training methodology it can reduce the costs and risks and has been adopted widely by the surgical community. A training system based on virtual simulation provides the trainees with flexible access to training sessions, whereas traditionally the training sessions are limited by the availability of patients and training posts. The most essential element of such a system is to model the behaviour of the tissues in a proper manner in order to produce convincing visual and haptic feedback to the user. Slender shapes represent a group of organs and tissues in the human body. Efficient simulation of such structures provides a useful framework which lays the foundation for virtual surgery applications involving such objects. Our work discussed in this paper focuses on modelling slender biological tissues which can be presented with a simple rod model.

In the human body, many tissues are of long, thin, rod-like shapes, and we can take the advantage of modelling them as one-dimensional entities to efficiently simulate their behaviours. Examples of such tissues include blood vessels, muscle fibres, small intestines, rectum, ligaments, tendons, and hair. Though the one 
dimensional rod-like object has been well studied in engineering, such as the analysis of the beam frames to improve the safety of structures in civil engineering, the calculation of the deformation for the above biological tissues remains a challenging task. For instance, the tissue may exhibit large deflexion that invalidates the algorithms which were derived based on the assumption of small deformation. The finite element method and mass spring method have been widely adopted in surgery simulation. They can provide accurate results, but they are generally inefficient in modelling the rod-like object if using volumetric discretization. Other classic methods based on the centreline of a rod, such as the Cosserat rod model [1], are designed to capture the global deformation, which provide little information about the local deformation. In order to capture the local deformation, the finite element method and the mass spring systems are often the only options which unfortunately are computationally expensive for real-time simulation.

In this paper, we propose to model the rod-like biological structure with two separate layers: global deformation and local deformation. To our knowledge, Barr [2] was the first who developed the idea in computer graphics, where global and local shape changes were dealt with separately. In our method, the Cosserat rod model is adopted to model the global deformation along the centre line, while the local deformation is characterised by the deformation of a given cross-section of the tissue. Large global deformation, such as twisting and bending, is well handled with the Cosserat rod model. The local stretch of the surface is then approximated with our local deformation module.

In general, the biological tissues are not isolated in the body. They are often attached to each other, which restricts their movement, i.e. they are usually subject to physical constraints. For instance, Raghupathi et al. [3] proposed to model the mesentery which connects the blood vessel and the intestine with a mass spring sheet. In this paper, the influence of such constraints can be integrated into the Cosserat model with an additional elastic energy item.

Our contributions in this paper are as follows: (1) we have developed a general framework of global and local deformations of long flexible rod-like biological tissues; (2) we have introduced a quasi-static Cosserat rod model for fast simulation of the global deformation of a rod with additional constraints; (3) we have established a local deformation model which performs in an efficient manner.

\section{Related Work}

There exists a large body of research in the area of soft tissue simulation $[4,5,6,7$, 8]. Due to the complexity of soft biological tissue, it is extremely challenging to simulate their natural behaviour accurately, especially when subject to interventions with surgical instruments.

Most of the current approaches are based on either the mass spring system $[9,10]$ or the finite element method $[4,11,12]$. The mass spring system uses discretized mass points in the space to represent the volume or surface. Such mass points are connected to each other with springs to capture the dynamics. The finite element method discretizes the object into many small elements, e.g. tetrahedrons. A problem is solved by finding the numerical solution of governing partial equations in an 
element-wise manner. The finite element method provides accurate results but it is computationally expensive.

Thin slender rod-like biological tissues have been studied in a wide range of disciplines. In computer animation, hair modelling has attracted a great deal of attention [13, 14], which can be used in character rigging. In applications of virtual surgery training, some researchers reported the development on modelling intestine with the mass spring system [3, 15]. In [16] a centreline model was introduced for long thin rod-like structures. By filtering out local changes of the cross-section shape, it greatly reduced the degrees of freedom of the problem and hence enabled the problem to be solved efficiently.

Cosserat rod can capture the large bending and twisting deformation of one dimensional rod-like object, which records the motion of points on the centreline and the rotation of a local coordinate frame attached to each individual point. It was used to model the deformation of cable like instrument in engineering [17]. Linn et al. [18] outlined a method to model rod deformation with Kirchhoff model (a special case of Cosserat model) for virtual reality applications. Several recent works [19, 20, 21, 22] in computer animation provided good reference of Cosserat rod model in detail. Despite its efficiency the Cosserat rod model's inability to handle local deformations often renders it unsuitable for high fidelity surgery training applications, as local deformation can provide useful feedback to the surgeons. In this paper, a quasi-static model of Cosserat rod is introduced to construct global deformations. Different from the previous approaches [20,21], our quasi-static model drops the dynamic items and simplifies the solving process to allow fast simulation. In order to overcome the drawback of lack of local deformations, in this paper we model the deformation of local regions separately, whose superposition with the global deformation provides convincing looking of the soft tissues.

\section{Cosserat Rod Mechanics}

The Cosserat rod model, which describes the dynamics of a long thin rod-like object, is a well established model in both traditional mechanics [1] and computer graphics $[20,21]$.

With the Cosserat rod model, a rod is represented as a space curve and its cross section is shrunk to one single point on the curve. Such curve is either selected as the neutral axis or as centroid axis (we will call this the curve centreline in the context). A local coordinate frame is attached to each point on the curve which indicates the position and orientation of the cross section. 


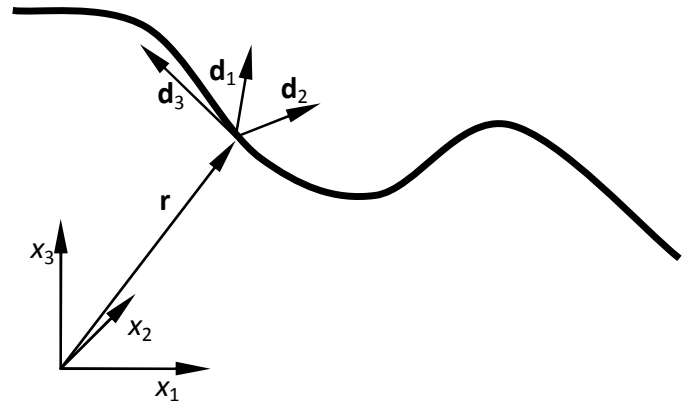

Fig. 1. Cosserat rod model.

As shown in Fig. 1, given $\mathbf{r}(s)$ as a point on the selected curve, where $s$ is the curve length ranging from 0 to $L$, we denote the coordinate frame by three unit vectors $\mathbf{d}_{1}, \mathbf{d}_{2}$ and $\mathbf{d}_{3}$. These three vectors are also named as directors, which are orthogonal to each other. In particular, $\mathbf{d}_{3}$ is defined to be along the tangent of the beam centreline, where the other two directors, $\mathbf{d}_{1}$ and $\mathbf{d}_{2}$, represent two orthogonal material lines in the cross-section perpendicular to $\mathbf{d}_{3}$.

$$
\mathbf{d}_{3} \times \frac{\partial \mathbf{r}}{\partial s}=0 ; \quad \mathbf{d}_{1} \times \mathbf{d}_{2}=\mathbf{d}_{3}
$$

With the definition of Darboux vector $\omega$ as

$$
\frac{\partial \mathbf{d}_{i}}{\partial s}=\boldsymbol{\omega} \times \mathbf{d}_{i} \quad i=1,2,3 ;
$$

we can write the torsion $(\tau)$ and the flexures $\left(\kappa_{1}, \kappa_{2}\right)$ of the rod as

$$
\boldsymbol{\omega}=\kappa_{1} \mathbf{d}_{1}+\kappa_{2} \mathbf{d}_{2}+\tau \mathbf{d}_{3}
$$

Apart from the bending and twisting deformation measured by torsion and flexures, a rod can have stretching and shearing deformations, which are described by another measure $\mathbf{v}$,

$$
\mathbf{v}=\frac{\partial \mathbf{r}}{\partial s}=v_{1} \mathbf{d}_{1}+v_{2} \mathbf{d}_{2}+v_{3} \mathbf{d}_{3}
$$

where $v_{1}$ and $v_{2}$ denote the shear deformation of the cross section which is the tilting of the cross-section, and $\left(v_{3}-1\right)$ denotes the stretch which is the length change of the beam. In practice, we often omit the influence of any shear deformation, that is $v_{1}=0$ and $v_{2}=0$.

In a surgical operation, the maneuver of the subject (the organ) is relatively slow, and a sudden change of speed rarely occurs. Therefore, we can omit the influence of the inertial force and model the deformation in a quasi-static fashion, unlike the previous work [20, 21] where the dynamic effect is considered. In our method, the overall potential energy is written as

$$
\Pi=U-W
$$


where $U=\frac{1}{2} \int\left[B_{1}\left(\kappa_{1}-\bar{\kappa}_{1}\right)^{2}+B_{2}\left(\kappa_{2}-\bar{\kappa}_{2}\right)^{2}+G J(\tau-\bar{\tau})^{2}+E A\left(v_{3}-1\right)^{2}\right] d s$ is the elastic energy of the rod and $W=\int \mathbf{f} \cdot(\mathbf{r}-\overline{\mathbf{r}}) d s$ is the work by the external force $\mathbf{f}$. Values with note ( $\left.{ }^{-}\right)$are initial state defined on the rest shape. $B_{1}$ and $B_{2}$ represent the bending stiffness, $E$ is the Young's modulus, $A$ is the area of cross section, $G$ is the shear modulus and $J$ is the polar moment of inertia of the cross sectional area. Minimization of the potential energy gives out the solution of a rod in equilibrium.

\subsection{Discretization of the Rod}

To find the numerical solution, we approximate a continual rod with $n+1$ vertices $\mathbf{x}_{0}$, $\mathbf{x}_{1}, \ldots, \mathbf{x}_{\mathrm{n}}$ and $n$ straight edge $\mathbf{e}_{0}, \mathbf{e}_{1}, \ldots, \mathbf{e}_{\mathrm{n}-1}$. The $i$ th edge $\mathbf{e}_{i}$ connected point $\mathbf{x}_{i}$ and $\mathbf{x}_{i+1}$. The local coordinate system is defined on each individual edge after discretization. For edge $\mathbf{e}_{i}$, we can have

$$
\mathbf{d}_{3}^{i}=\frac{\mathbf{e}_{i}}{\left\|\mathbf{e}_{i}\right\|} ; \quad \mathbf{d}_{1}^{i} \times \mathbf{d}_{2}^{i}=\mathbf{d}_{3}^{i}
$$

With the above notation, we can define the discrete curvature and torsion on vertex $\mathbf{x}_{i}$ as:

$$
\begin{aligned}
\kappa_{1}^{i} & =\frac{2\left(\mathbf{d}_{3}^{i-1} \times \mathbf{d}_{3}^{i}\right)}{\left(\left\|\mathbf{e}_{i-1}\right\|+\left\|\mathbf{e}_{i}\right\|\right)^{2}} \cdot\left(\left\|\mathbf{e}_{i-1}\right\| \mathbf{d}_{2}^{i-1}+\left\|\mathbf{e}_{i}\right\| \mathbf{d}_{2}^{i}\right) \\
\kappa_{2}^{i} & =-\frac{2\left(\mathbf{d}_{3}^{i-1} \times \mathbf{d}_{3}^{i}\right)}{\left(\left\|\mathbf{e}_{i-1}\right\|+\left\|\mathbf{e}_{i}\right\|\right)^{2}} \cdot\left(\left\|\mathbf{e}_{i-1}\right\| \mathbf{d}_{1}^{i-1}+\left\|\mathbf{e}_{i}\right\| \mathbf{d}_{1}^{i}\right) \\
\tau^{i} & =\frac{2\left(\mathbf{d}_{1}^{i-1} \times \mathbf{d}_{1}^{i}\right)}{\left(\left\|\mathbf{e}_{i-1}\right\|+\left\|\mathbf{e}_{i}\right\|\right)^{2}} \cdot\left(\mathbf{e}_{i-1}+\mathbf{e}_{i}\right)
\end{aligned}
$$

Here the directors $\mathbf{d}_{1}^{i}, \mathbf{d}_{2}^{i}, \mathbf{d}_{3}^{i}$ are defined on the $i$ th edge and the curvature $\left(\kappa_{1}^{i}, \kappa_{2}^{i}\right)$ and torsion $\tau^{i}$ are defined on the $i$ th vertex. And the stretch on vertex $\mathbf{x}_{i}$ is defined as

$$
v_{3}^{i}=\frac{\left\|\mathbf{e}_{i-1}\right\|+\left\|\mathbf{e}_{i}\right\|}{\left\|\overline{\mathbf{e}}_{i-1}\right\|+\left\|\overline{\mathbf{e}}_{i}\right\|}
$$

with note $\left(^{-}\right)$denoting initial state defined on the rest shape.

\subsection{Biological Tissues with Constraints}

In real anatomy, rod-like structures are embedded into surrounding tissues or fixed at certain position with ligaments and/or fat tissues. For example, the rectum is surrounded by the pelvic skeletal structure. To remove a low rectal cancer requires cutting the rectum near the anal verge and the surgeon has to separate the colon near the anal verge around the perimeter. To model the effect of such constraints, we added springs to the Cosserat rod model as shown in Fig. 2. 


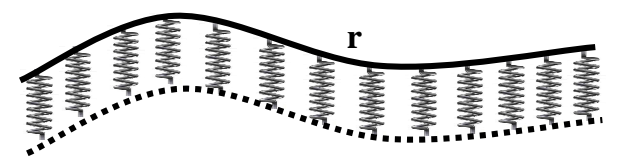

Fig. 2. Model the constraint of surrounding tissues.

With the additional springs, we can rewrite the elastic energy $U$ in equation (5) as $U=\frac{1}{2} \int\left[B_{1}\left(\kappa_{1}-\bar{\kappa}_{1}\right)^{2}+B_{2}\left(\kappa_{2}-\bar{\kappa}_{2}\right)^{2}+G J(\tau-\bar{\tau})^{2}+E A\left(v_{3}-1\right)^{2}+K(\|\mathbf{r}-\overline{\mathbf{r}}\|)^{2}\right] d s$

where the last item describes the contribution of the spring constraints. $K$ is the spring stiffness distribution of the constraints.

If an operation involves separating part or all of the organ from its supporting tissue, we can model the effect of cutting by altering the distribution of stiffness $K$. $K(s)$ equals to 0 means that the rod has no connection to its surrounding tissue at point $\mathbf{r}(s)$.

Fig. 3 shows results of a uniform elastic rod under gravity, fixed on one end. The top is the result for rod without springs and the bottom is the result for rod with spring constraints.

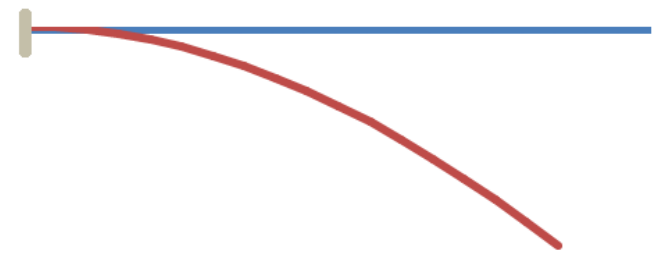

(a)

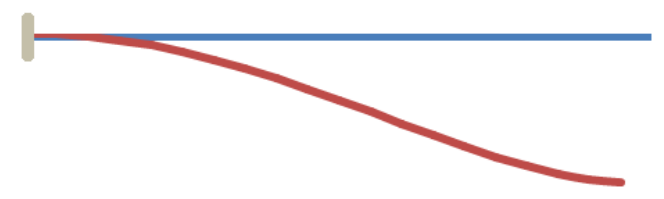

(b)

Fig. 3. Deformation of a rod without or with spring constraint.

\section{$4 \quad$ Local Deformation Modelling}

The Cosserat rod model represents the motion of centreline and rotation of cross section, which reflects the global changes. However, as the cross section has shrunk 
into one point in geometry, the shape of cross section, which relates to the local deformation caused by the intervention of surgical instruments, is missing from the Cosserat model.

The reaction force on the instrument can be calculated with the Cosserat rod model by consideration of the global deformation along the centreline. But the local deformation of a soft tube-like structure cannot be ignored in some cases. For example, in the resection operation removing a rectum cancer, poking or pushing the bowel causes visible deformation of the tube wall. Previously, such deformation had to be modelled with either the mass spring system or the finite element method, which increases the complexity of computation and modelling. Furthermore, when the geometric mesh of the mass spring system or the finite element method is coarse or distorted, the local deformation may be filtered out and not shown correctly.

Here, we select a curve $C$ resembling the cross-section at intervention point and deform it to create a local deformation. Being a manifold of a circle, the curve $C$ can be parameterised with angle $\theta$ from 0 to $2 \pi$ as $\mathbf{R}(\theta)$. We assume that the deformed $C$ takes an in-plane deformation only. Therefore the deformed shape of the curve is characterised by minimising the potential energy, $\Pi=U-W$.

The elastic energy is defined as

$$
U=\frac{1}{2} \int\left[B(\kappa-\bar{\kappa})^{2}+E A\left(v_{3}-1\right)^{2}\right] \frac{d\|\mathbf{R}\|}{d \theta} d \theta
$$

where the first item presents the energy of bending, and the second item denotes that of stretching. Only one bending item appears in an in-plane deformation and there is no twisting. We write the work by external forces as follows,

$$
W=\mathbf{F} \cdot\left(\mathbf{R}_{f}-\overline{\mathbf{R}}_{f}\right)-\int \frac{\mathbf{F}}{L_{c}} \cdot(\mathbf{R}-\overline{\mathbf{R}}) \frac{d\|\mathbf{R}\|}{d \theta} d \theta
$$

where values with note ( $)$ are initial values defined on the rest shape, $\mathbf{F}$ is the projection of the exerted force in the cross section at point $\mathbf{R}_{f}$, and a body force of $-\mathbf{F} / L_{c}$ is distributed along the curve to balance it, where $L_{c}$ is the length of curve $C$. An additional constraint is added which ensures the mass centre of the curve unchanged during the deformation.

Once curve $C$ is deformed, its deformation is propagated to its neighbouring mesh points. Firstly, before deforming the vertices of the edges which $C$ comes across are selected (see Fig. 4(a)). For one of the selected vertices, p, we can find the closest point on the curve as $\overline{\mathbf{R}}_{p}$ with distance $d$. Vertex $\mathbf{p}$ is moved to the new position $\mathbf{p}^{\prime}$ with

$$
\mathbf{p}^{\prime}=\mathbf{p}+(1-d / h)^{2}\left(\mathbf{R}_{p}-\overline{\mathbf{R}}_{p}\right)
$$

where $h$ is a control parameter, which we set to $0.56 \sqrt{A} . A$ is the area of crosssection $C$. New points are then added to propagate the deformation further. These newly added points have edge connections to the previous selected points (see Fig. 4(b)). Given a new added point $\mathbf{p}$ which connected to the previous selected $k$ points $\mathbf{p}_{1}, \mathbf{p}_{2} \ldots \mathbf{p}_{k}$, its new position is given as

$$
\mathbf{p}^{\prime}=\mathbf{p}+\frac{1}{k} \sum_{i=1}^{k}\left(1-e_{i} / h\right)^{2}\left(\mathbf{p}_{i}^{\prime}-\mathbf{p}_{i}\right)
$$


where $e_{i}$ is the edge length of $\mathbf{p}_{i}$ and $\mathbf{p}$. This process then iterates until $\left\|\mathbf{p}^{\prime}-\mathbf{p}\right\|$ is less than a given threshold $0.01 \sqrt{A}$.

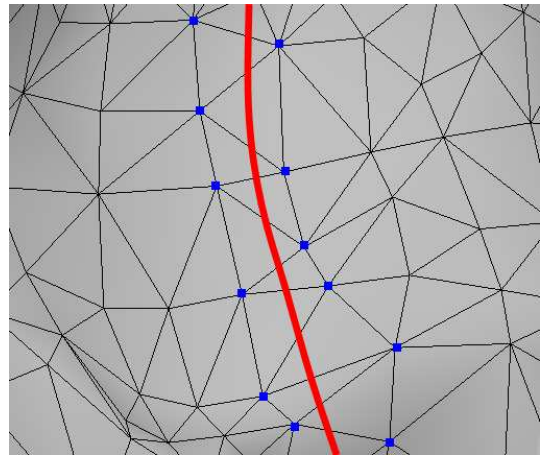

(a)

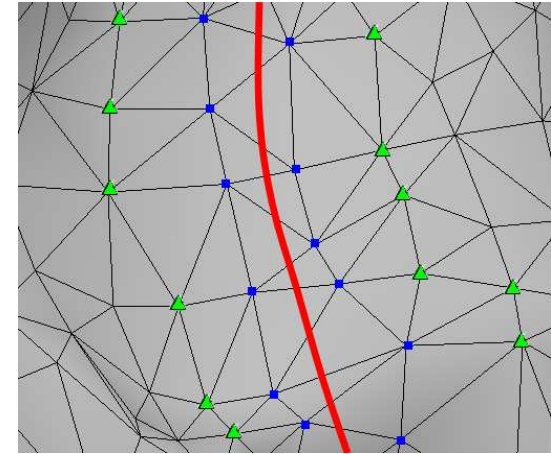

(b)

Fig. 4. (a) Selecting mesh vertices (blue squares) which the curve $C$ (red curve) comes across. (b) New added vertices (green triangles) which connect to the previous selected vertices (blue squares).

\section{$5 \quad$ Results}

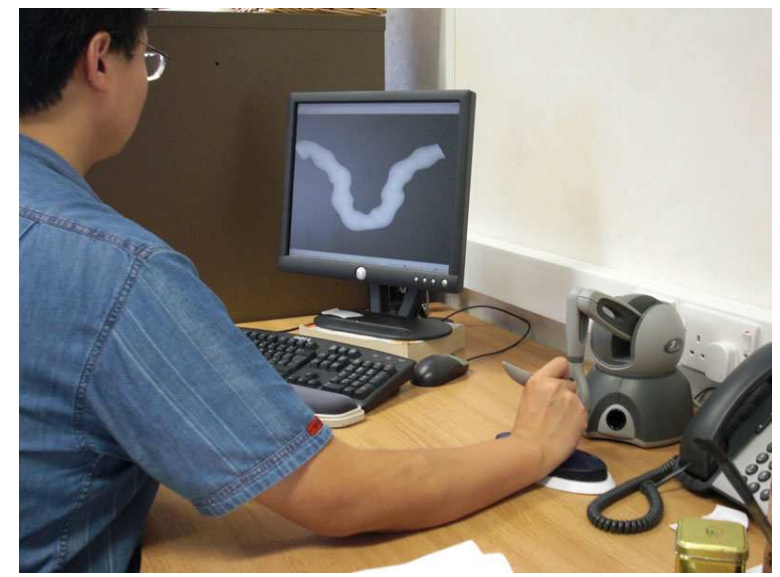

Fig. 5. A Phantom Omni device was used to get haptic output.

A Phantom Omni device is used to control the drag and poke actions at a point on the rod-like structure. As shown in Fig. 5, the user can input the 3D coordinates of a selected point on the mesh to mimic the intervention of the instrument in surgery and the Phantom Omni device provides force feedback according to the input. 


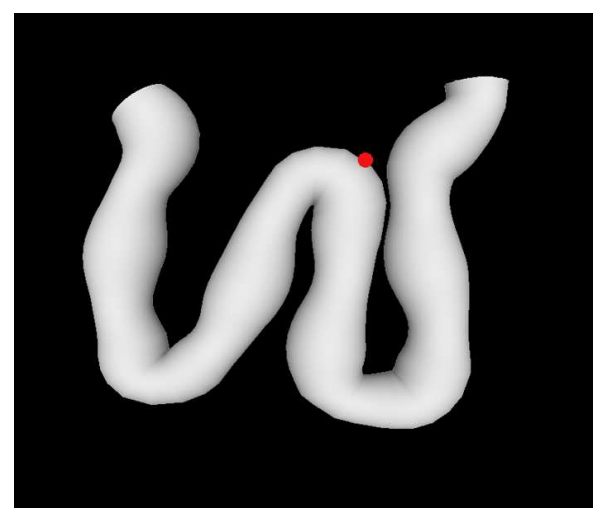

(a)

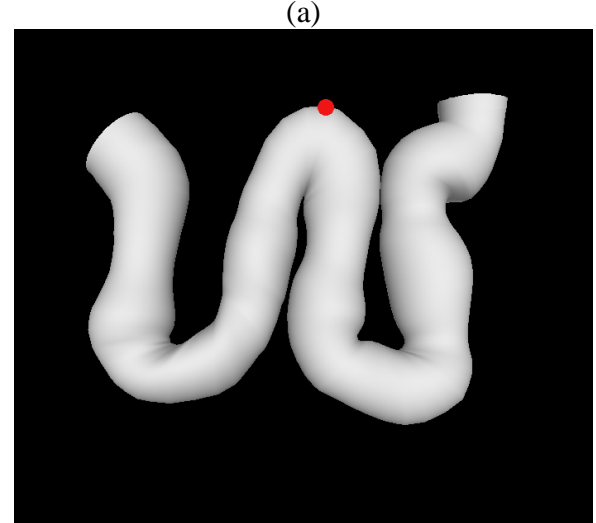

(b)

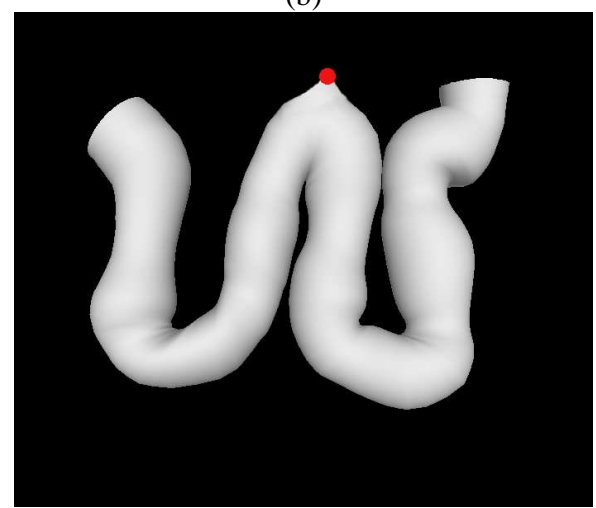

(c)

Fig. 6. (a) a section of intestine before deformation; (b) the section of intestine deformed globally when being dragged upwards; (c) a local deformation added after global deformation.

Fig. 6 shows the results without (Fig. 6(b)) or with (Fig. 6(c)) local deformation, which are simulation of a pull operation on a section of a bowel structure. It is observed that the local deformation enhanced the visual fidelity in the simulation. 


\section{Conclusions}

We have presented a method to model one dimensional rod-like biological tissues, which handles both global and local deformations plausibly in an efficient manner. Our strategy is to formulate them with two separate layers. Global deformations of such tissues like bending and twisting are simulated with the Cosserat rod model, while local deformations are handled separately. The cross-section where the $\mathrm{drag} /$ poke action is exerted is extracted to generate local deformations, which are also represented with an enclosed curve subject to the given loads. The approximated local deformation propagates to the neighbouring vertices in a geometric manner. Our approach provides a good balance between visual fidelity and computational efficiency. However, if higher accuracy of the local deformation is necessary to an application, one can always use a more accurate and a slower approach, such as nonlinear finite element computation.

The main benefit of using a one dimensional entity to present the physics of the object is efficiency. Fewer degrees of freedom are required to discretize a curve than a surface or a volume in order to capture the change of physical variables. When modelling a slender structure, our approach is more desirable than the traditional finite element method or mass spring system with volumetric discretization.

We have not considered the influence of self-collision in this implementation. It is possible to include known contact algorithms, e.g. the method in [3], into the prototype. In the future, we would also like to extend this work to consider the nonlinear material properties and to model the cutting operation, which requires changes of the mesh topology locally as well as changes of the physical properties of the object accordingly.

\section{References}

1. Antman, S.: Nonlinear Problems of Elasticity. New York: Springer-Verlag.(1995)

2. Barr, A. H.: Global and local deformations of solid primitives, In: SIGGRAPH'84, 21-30. (1984)

3. Raghupathi, L., Grisoni, L., Faure, F., Marchal, D., Cani, M., and Chaillou, C.: An intestinal surgery simulator: real-time collision processing and visualization. IEEE Trans. Visual. Comput. Graphics. 10(6), 708-718. (2004)

4. Bro-nielsen, M.: Finite element modeling in surgery simulation, Proc. of the IEEE, 86(3): pp.490-503.(1998)

5. Cotin, S., Delingette, H. and Ayache, N.: Real-time elastic deformations of soft tissues for surgery simulation, IEEE Trans. Visual. Comput. Graphics. 5(1), 62-73. (1999)

6. Delingette, H., and Ayache, N.: Hepatic surgery simulation, Commun. ACM, 48(2), 3136. (2005)

7. Kim, Y., Lee, K., and Kim, W.: 3D virtual simulator for breast plastic surgery. Comput. Animat. Virtual Worlds 19(3-4), 515-526. (2008)

8. Comas, O., Taylor, Z. A., Allard, J., Ourselin, S., Cotin, S., and Passenger, J: Efficient nonlinear FEM for soft tissue modelling and its GPU implementation within the open source framework SOFA. In: Bello F. and Edwards, P. (eds.) ISBMS '08. LNCS. vol. 5104, 28-39. Springer-Verlag, Berlin, Heidelberg. (2008) 
9. Mollemans, W., Schutyser, F., Cleynenbreugel, J. and Suetens, P.: Fast soft tissue deformation with tetrahedral mass spring model for maxillofacial surgery planning systems. In: Barillot, C., Haynor, D., and Hellier P. (eds.) MICCAI 2004. LNCS. vol. 3217, 371-379. Springer-Verlag, Berlin, Heidelberg. (2004)

10. Hong, M., Jung, S., Choi, M., and Welch, S. W.: Fast volume preservation for a massspring system. IEEE Comput. Graph. Appl. 26(5), 83-91.(2006)

11. Bathe, K. J.: Finite Element Procedures, 2nd edition, London: Prentice-Hall. (1996)

12. $\mathrm{Wu}, \mathrm{W}$. and Heng, P. A.: A hybrid condensed finite element model with GPU acceleration for interactive 3D soft tissue cutting. Comput. Animat. Virtual Worlds 15(3-4), 219227.(2004)

13. Bertails, F., Audoly, B., Cani, M., Querleux, B., Leroy, F., and Lévêque, J.: Super-helices for predicting the dynamics of natural hair. In: SIGGRAPH'06, 1180-1187.(2006)

14. Ward, K., Bertails, F., Kim, T., Marschner, S. R., and Cani, M.: A Survey on hair modeling: styling, simulation, and rendering. IEEE Trans. Visual. Comput. Graphics, 13(2), 213-234. (2007)

15. France, L., Lenoir, J., Angelidis, A., Meseure, P., Cani, M., Faure, F., and Chaillou, C.: A layered model of a virtual human intestine for surgery simulation, Med. Image Anal., 9(2), 123-132. (2005)

16. Huang, P., Gu, L., and Zhang, S.: Real-time simulation for global deformation of softtissue using deformable centerline and medial representation. In: Harders, M. and Székely, G. (eds.) ISBMS 2006, LNCS, vol. 4072, 67- 74, (2006)

17. Grégoire, M. and Schömer, E.: Interactive simulation of one-dimensional flexible parts. Comput. Aided Des., 39(8), 694-707.(2007)

18. Linn, J., Stephan, T., Carlsson, J. and Bohlin, R.: Fast simulation of quasistatic rod deformations for VR applications. Progress in Industrial Mathematics at ECMI 2006, Part 2, 247-253. (2007)

19. Pai, D.: Strands: Interactive simulation of thin solids using Cosserat models, Comp. Graph. Forum, 21(3), 347-352. (2002)

20. Spillmann, J., Teschner, M.: CoRdE: Cosserat rod elements for the dynamic simulation of one-dimensional elastic objects, In: SCA'07, 63-72. (2007)

21. Bergou, M., Wardetzky, M., Robinson, S., Audoly, B., and Grinspun, E.: Discrete elastic rods. In SIGGRAPH'08, Article 63. (2008)

22. Chang, J., Shepherd, D., Zhang, J.J.: Cosserat-beam-based dynamic response modelling, Comput. Anim. Virtual Worlds, 18(4-5), 429-436. (2007) 